\title{
Nanofibrous gelatine scaffolds integrated with nerve growth factor-loaded alginate microspheres for brain tissue engineering
}

\author{
Melda Büyüköz ${ }^{1}$, Esra Erdal ${ }^{2}$ and Sacide Alsoy Altinkaya ${ }^{3 *}$ \\ ${ }^{1}$ Department of Biotechnology and Bioengineering, Izmir Institute of Technology, Turkey \\ ${ }^{2}$ Izmir Biomedicine and Genome Institute, Dokuz Eylul University, Turkey \\ ${ }^{3}$ Department of Chemical Engineering, Izmir Institute of Technology, Turkey
}

\begin{abstract}
Neural regeneration research is designed in part to develop strategies for therapy after nerve damage due to injury or disease. In this study, a new gelatine-based biomimetic scaffold was fabricated for brain tissue engineering applications. A technique combining thermally induced phase separation and porogen leaching was used to create interconnected macropores and nanofibrous structure. To promote tissue regeneration processes, the scaffolds were integrated with nerve growth factor (NGF)-loaded alginate microspheres. The results showed that nanofibrous matrix could only be obtained when gelatine concentration was at least $7.5 \%(w / v)$. The scaffold with a modulus value $(1.2 \mathrm{kPa})$ similar to that of brain tissue $(0.5-1 \mathrm{kPa})$ was obtained by optimizing the heat treatment time, macropore size and gelatine concentration. The encapsulation efficiencies of NGF into $0.1 \%$ and $1 \%$ alginate microspheres were $85 \%$ and $100 \%$, respectively. The release rate of NGF from the microspheres was controlled by the alginate concentration and the poly(L-lysine) coating. The immobilization of the microspheres in the scaffold reduced burst release and significantly extended the release period. The nanofibrous architecture and controlled release of NGF from the microspheres induced neurite extension of PC12 cells, demonstrating that the released NGF was in an active form. The results suggest that the scaffolds prepared in this study may have potential applications in brain tissue engineering due to topologic and mechanical properties similar to brain tissue and pore structure suitable for cell growth and differentiation. Copyright (C) 2016 John Wiley \& Sons, Ltd.
\end{abstract}

Received 19 December 2015; Revised 30 September 2016; Accepted 9 November 2016

Keywords gelatine scaffold; nanofibre; alginate microspheres; nerve growth factor; controlled delivery; brain tissue engineering

\section{Introduction}

The regeneration capacity of neurons and axonal regrowth in the brain following an injury or disease is limited due to the presence of inhibitory molecules and lack of neurotropic factors at the injured site (Schmidt and Leach 2003). To promote tissue regeneration, cellbased therapies have been used via transplantation of mesenchymal stromal cells, neural stem/progenitor cells, embryonic stem cells, induced pluripotent stem cells into the injured and/or diseased sites. However, this strategy was not found to be successful due to low cell survival, uncontrolled differentiation and ineffective cell integration into the host tissue (Tam et al., 2014). Scaffolds play a crucial role as an artificial microenvironment to support survival of transplanted cells, facilitate cell regeneration and prevent further damage to adjacent tissue. Potential application areas of 3D scaffolds in brain tissue engineering include an in vitro nerve tissue stem cell niche (Ma et al., 2014; Mahmood et al., 2014; Qu et al., 2009, 2011), cell culture

*Correspondence to: Sacide Alsoy Altinkaya, Department of Chemical Engineering, Izmir Institute of Technology, Gulbahce Koyu, 35430 Urla, Izmir, Turkey. E-mail: sacidealsoy@iyte.edu.tr matrix for the investigation of neurodegenerative disease, treatment mechanism and regeneration of brain tissue (D’Avanzo et al., 2015; Kim et al., 2015; Simão et al., 2015), implantable drug delivery system in brain cancer (Mak et al., 1995; Vukelja et al., 2007; Westphal et al., 2003), and neurodegenerative diseases. Most of these studies are in vitro and still in their early stages and more investigation including both in vitro and in vivo studies are needed before moving onto clinical studies. However, the utilization of scaffolds in repairing damaged brain tissue is proposed as a feasible treatment option (Spires et al., 2004). Hydrogels are a commonly used type of scaffold for brain tissue engineering applications. They can be injected into a lesion site and enable the filling of irregular cavities with a minimally invasive procedure (Pettikiriarachchi et al., 2010). However, their high water sorption capacity adversely affects their mechanical integrity, facilitating fast degradation, resulting in collapse of the structure. As a result, cell reorganization in hydrogels occurs in a microenvironment without physical constraints, preventing formation of large-sized neural networks (Tang-Schomer et al., 2014). A silkfibroin based three-dimensional (3D) scaffold allowed long-term neural tissue viability up to 9 weeks and showed better performance than collagen-based 
hydrogels (Tang-Schomer et al., 2014) due to the increased degree of neural clustering in the scaffold structure as opposed to dispersal of neurons in the hydrogel. Self-assembling peptide nanofiber scaffolds manufactured from oligopeptides or amphiphilic molecules were also applied to brain lesions (Pettikiriarachchi et al., 2010). Although they have high porosity, tissue-like water content and enhanced signalling, resulting from the presence of bioactive peptide sequences, they suffer from weak mechanical properties due to their high water content and are susceptible to rapid enzymatic degradation (Pettikiriarachchi et al., 2010). Studies over the last two decades have shown that $3 \mathrm{D}$ macroporous nanofibrous (NF) scaffolds represent a promising approach for enhancement of neural network formation and ingrowth (Ma and Choi 2001; Wei et al., 2007b).

The idea of combining macroporous NF scaffold with protein loaded microspheres was first proposed by $\mathrm{Ma}$ (2004) and the advantages of these biomimetic scaffolds were shown in cartilage and bone tissue engineering (Wei and Ma 2008; Wei et al., 2007a). In brain tissue engineering, NF scaffolds were produced from a variety of synthetic polymers using an electrospinning technique (Pettikiriarachchi et al., 2010); however, 3D scaffolds featuring macroporous NF structure and incorporated with neurotrophic factor-loaded microspheres have not yet been investigated. This study therefore aimed to prepare gelatine-based NF scaffolds integrated with nerve growth factor (NGF)-loaded alginate microspheres for brain tissue engineering applications. The major design criterion for optimizing preparation conditions was the elastic modulus of native brain tissue, since colonization, migration, differentiation of neural cells (Leipzig and Shoichet 2009; Saha et al., 2008) and altered neurite formation and trajectory (Balgude et al., 2001; Jiang et al., 2008) have been found to be influenced by matrix stiffness. In addition to mechanical and structural properties, biodegradation rate of the scaffold is also an important issue for brain tissue engineering applications. It becomes necessary to have a long-term scaffold especially for large lesions. Gelatine was selected as the scaffold material since it is a derivative of collagen and it has the integrin-binding domain (arginine, glycine and aspartic acid) sequence (Chen et al., 2006) in its structure, which enhances adhesion of cells. Its degradation rate can be easily adjusted to match desired rate by adjusting degree of crosslinking. A strong polyanion, alginate, has been chosen for loading NGF through mainly electrostatic interactions. The method developed by Ciofani et al., (2008) has been modified to achieve increased microsphere yield (ratio of mass of particles obtained to the mass of polymer used in the emulsion) and consequently high NGF loading into alginate microspheres. The scaffolds were prepared by combining thermally induced phase separation and porogen leaching processes. As an innovative approach, the same reaction was used for crosslinking the scaffold and immobilizing alginate microspheres to the scaffold using 1-ethyl-3-(3-dimethyl-aminopropyl) carbodiimide
(EDC)-N-hydroxysuccinimide (NHS) coupling reagents. The influences of manufacturing conditions on the structure and mechanical properties of the scaffolds were examined. In addition, the in vitro performance of the scaffold on the proliferation and differentiation of the model cell line, PC12, was investigated. Overall, our 3D NF scaffolds, which have tunable mechanical properties and allow for sustained delivery of NGF, can become a potential therapeutic candidate for brain tissue engineering applications, especially in traumatic brain injuries. To the best of our knowledge, this study is the first which develops biomimetic NF scaffold for brain tissue engineering applications.

\section{Materials and methods}

\subsection{Materials}

Paraffin with a melting point of $53-57^{\circ} \mathrm{C}$, gelatine (type $\mathrm{B}$, from bovine skin), EDC, NHS, (2-(N-morpholino) ethanesulfonic acid) hydrate, alginate (medium viscosity), sodium chloride, sodium citrate, poly-L-lysine with a molecular weight 30,000-70,000, Tween 80 and Span 80 were all supplied by Sigma-Aldrich (St Louis, MO, USA). Acetone, 1,4-dioxane, n-hexane, cyclohexane, ethanol, isooctane, calcium chloride, and mouse-NGF were purchased from Merck (Darmstadt, Germany). Poly(vinyl alcohol) with a molecular weight of 25,000 was purchased from Polyscience (Warrington, PA, USA). RPMI 1640, horse serum, fetal bovine serum, L-glutamine, antibiotic (penicillin, streptomycin), and 2,3-bis-(2-methoxy-4-nitro5-sulfophenyl)-2H-tetrazolium-5-carboxanilide (XTT) were obtained from Biological Industries (Kibbutz Beit Haemek, Israel), and glutaraldehyde used was from Alfa-Easer (Karlsruhe, Germany).

\subsection{Preparation of NF scaffold}

Macroporous NF gelatine scaffolds were prepared using a previously developed protocol (Liu and Ma 2009). Paraffin spheres were prepared as pore-forming agent with the method suggested by Ma and Choi (2001). Briefly, spheres with different sizes (250-425 $\mu \mathrm{m}$ and 425-600 $\mu \mathrm{m}$ ) were added to a custom made Teflon mould (with diameter of $1.7 \mathrm{~cm}$ and depth of $1.5 \mathrm{~cm}$ ) and the top surface was levelled. The mould was then heated at $37^{\circ} \mathrm{C}$ to obtain interconnected spheres. Gelatine was dissolved in $50 / 50(v / v)$ ethanol-water solution at $45^{\circ} \mathrm{C}$ and $0.35 \mathrm{ml}$ of this solution was cast onto the paraffin sphere assemblies. The gelatine solution in the paraffin assembly was phase separated at $-80^{\circ} \mathrm{C}$ for $5 \mathrm{~h}$. Next, the scaffolds were immersed in cold ethanol $\left(-18^{\circ} \mathrm{C}\right)$ and transferred into 1,4-dioxane for $24 \mathrm{~h}$ for solvent exchange. After freeze-drying, gelatine/paraffin composites were soaked in hexane at $37^{\circ} \mathrm{C}$ for 3 days, then cyclohexane was used to exchange hexane in the scaffold. As a final step, the scaffolds were freeze-dried for 4 days. 


\subsection{Preparation of alginate microspheres}

Alginate microspheres were prepared by external ionic gelation and reticulation of alginate with calcium ions in water-in-oil emulsion system. For this, a method proposed by Ciofani et al., (2008) was modified. First, iso-octane was mixed with Tween 80 and Span 80, then alginate solution was added to this mixture and homogenized at 15,000 revolutions/min (rpm; IKA, Ultra-turrax). During homogenization, $2 \mathrm{ml}$ of $100 \% \mathrm{CaCl}_{2}(w / v)$ was added drop wise to crosslink alginate. After additional homogenization for $30 \mathrm{~s}$, the system was allowed to settle and the supernatant was removed. Next, the microspheres were stabilized with an acetone and water mixture [1:10 $(v / v)]$ at $1900 \mathrm{~g}$ for $5 \mathrm{~min}$. To prepare protein-loaded alginate microspheres, $100 \mu \mathrm{l}$ of $10 \mu \mathrm{g} / \mathrm{ml}$ of NGF was mixed with alginate solution and this mixture was added to the iso-octane, Tween 80 and Span 80 mixture. The sizes of microspheres were measured with a phase contrast microscope by dispersing the microspheres in water. To control release rate of NGF, alginate microspheres were first coated with poly(L-lysine) (PLL) and then with the alginate. In this step, harvested microspheres were suspended in $100 \mu \mathrm{g} / \mathrm{ml}$ of PLL solution for $30 \mathrm{~min}$. After removing the PLL solution by centrifugation, the alginate solution was added to microspheres, shaken for $30 \mathrm{~min}$, then samples were washed with deionized water and $20 \% \mathrm{CaCl}_{2}$ was added to crosslink alginate. Finally, $\mathrm{CaCl}_{2}$ solution was removed by centrifugation, and microspheres were washed, and these steps were repeated in triplicate.

\subsection{Chemical crosslinking of scaffold and the attachment of alginate microspheres to the gelatine scaffold}

EDC with NHS were used as cross linkers of gelatine scaffolds with NHS to EDC mole ratio of 0.2 . In the reaction, the molar ratio of $\mathrm{EDC}$ to free $\mathrm{COOH}$ groups in gelatine was taken into consideration and this ratio was fixed as 2:1 (as described in Supporting Information).
The reaction was carried out in 2-(N-morpholino) ethanesulfonic acid hydrate buffer $(\mathrm{pH} 5.3,0.05 \mathrm{M})$ at $+4^{\circ} \mathrm{C}$ for $24 \mathrm{~h}$. Acetone/water with a ratio of $90 / 10(v /$ v) was used instead of pure water to protect the NF structure (Liu and Ma 2009).

In the presence of EDC, amide linkages between lysine residue of gelatine and the carboxylate moieties of alginate polysaccharide is formed on polymer backbone (Hermanson 1996) as shown in Scheme 1. Therefore, EDC was used in this study, not only for crosslinking the scaffold but also for attaching the alginate spheres to the gelatine matrix. For this purpose, $18 \mathrm{mg}$ of $1 \%$ and $1.8 \mathrm{mg}$ of $0.1 \%$ of alginate microspheres were suspended into $100 \mu \mathrm{l}$ of crosslinking solution and $50 \mu \mathrm{l}$ of this solution was added onto two sides of the disc shaped scaffold with a diameter of $16 \mathrm{~mm}$ and height of $2 \mathrm{~mm}$. After waiting for $10 \mathrm{~min}$ for microspheres attachment, the scaffolds were kept in crosslinking solution for $24 \mathrm{~h}$ at $+4^{\circ} \mathrm{C}$, finally washed with deionized water three times and freeze-dried for 3 days.

The amount of alginate microspheres attached to the gelatine scaffold was determined using the phenol sulfuric acid assay (Gu et al., 2004). Scaffolds integrated with microspheres were taken into $1 \mathrm{ml}$ water, and $1 \mathrm{ml}$ of $5 \%(w / v)$ phenol and $5 \mathrm{ml}$ of concentrated sulfuric acid were added into the tube. The mixture was vortexed and kept at room temperature for $15 \mathrm{~min}$. The sample was then transferred to a 96-well plate, and absorbance was measured at $488 \mathrm{~nm}$. In this experiment negative and positive controls were the same size of plain scaffold and alginate microspheres equal in number to the free particles in solution that was added to the scaffold, respectively. Microspheres attached to the scaffolds were calculated using:

$$
\text { \%Attachment }=\left(\mathrm{A}_{1} / \mathrm{A}_{0}\right) \times 100
$$

where $A_{0}$ is absorbance value of positive control and $A_{1}$ is absorbance value of microsphere attached gelatine scaffold after subtracting absorbance of plain scaffold (negative control).

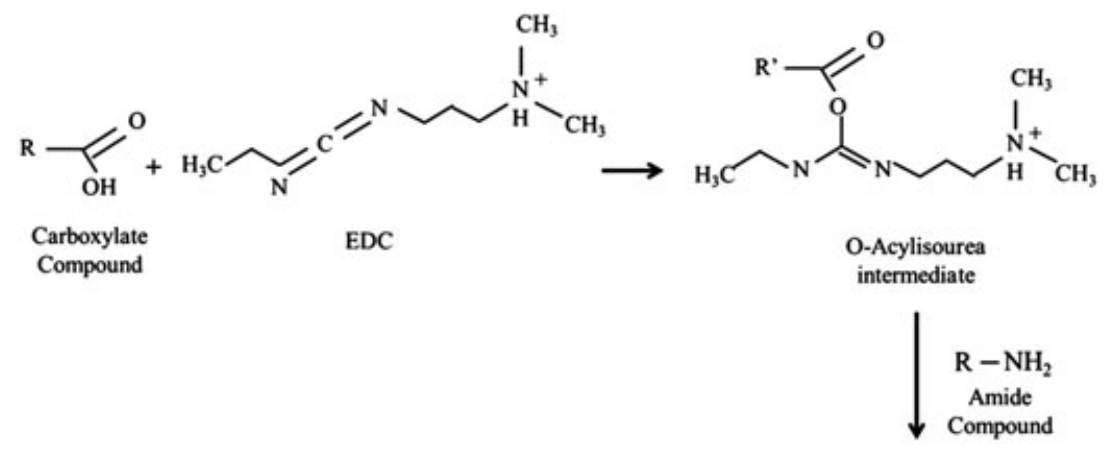

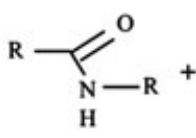

Amide Bond<smiles>CCNC(=O)NCCC[N+](C)C</smiles> 


\subsection{Characterization of NF scaffold}

\subsubsection{Surface morphology examination}

The morphology of the NF scaffolds and alginate microspheres were examined by scanning electron microscopy (SEM) on a Philips XL-30SFG model. The samples were coated with gold using a Magnetron Sputter Coating Instrument.

\subsubsection{Mechanical test}

A unidirectional compression test was performed on hydrated scaffold samples using a mechanical tester (Schimadzu AG-I $5 \mathrm{kN}$ ). All samples were circular discs $16 \mathrm{~mm}$ in diameter and $2 \mathrm{~mm}$ in thickness. Before the mechanical test, scaffolds were kept in phosphatebuffered saline $(\mathrm{pH}=7.4)$ for $24 \mathrm{~h}$. At least five specimens were tested for each sample. Values for the compressive modulus of elasticity were recorded at a strain of $5 \%$ and the average values with standard deviations were reported.

\subsection{Determination of NGF loading and release from alginate microspheres}

Alginate microspheres were transferred to a siliconized tube and $1 \mathrm{ml}$ of $3 \%(w / v)$ of Na-citrate solution was added. The tube was rotated at $300 \mathrm{rpm}$ in an orbital shaker. $\mathrm{Ca}^{2+}$ ions in the microspheres were exchanged with $\mathrm{Na}^{+}$ions destroying the structure of the particles. Next, the tube was centrifuged at $1900 \mathrm{~g}$ for $5 \mathrm{~min}$ and the supernatant was kept at $-20^{\circ} \mathrm{C}$. Encapsulation efficiency was calculated as follows:

Encapsulation efficiency $(\%)=\frac{\text { Loaded amount of NGF }}{\text { Initial amount of NGF }} \times 10$ 100

For the release study, alginate microspheres and microsphere attached scaffolds were put into a $1 \mathrm{ml}$ starvation medium (RPMI 1640 and 1\% horse serum) and shaken at $180 \mathrm{rpm}$ at $37^{\circ} \mathrm{C}$. At the end of different time intervals, the tubes were centrifuged at $1900 \mathrm{~g}$ for $5 \mathrm{~min}$ and the supernatant was analysed to determine the concentration of released NGF. Fresh starvation medium ( $1 \mathrm{ml}$ ) was added onto the microspheres to continue release studies. The amount of NGF loaded into microspheres or released from microspheres was determined with mouse NGF enzyme-linked immunosorbent assay kit (Boster Immunoleader).

\subsection{In vitro cell culture}

Rat adrenal pheochromocytoma (PC12) cell line was kindly donated by the Department of Medical Biochemistry at Dokuz Eylul University. Cells were cultured in $0.1 \mathrm{mg} / \mathrm{ml}$ PLL-coated $75-\mathrm{cm}^{2}$ flask in RPMI 1640 medium supplemented with 10\% horse serum, 5\% fetal bovine serum, 1\% L-glutamine, $1 \%$ penicillin/streptomycin $(100 \mathrm{U} / \mathrm{ml}$ penicillin and $100 \mu \mathrm{g} / \mathrm{ml}$ streptomycin) and incubated at $37^{\circ} \mathrm{C}$ in $5 \%$ $\mathrm{CO}_{2}$ and $95 \%$ air.

\subsubsection{Proliferation of $P C_{12}$ cells on macroporous NF gelatine scaffold integrated with empty alginate microspheres}

The proliferation behaviour of PC12 cells was investigated with 2, 3-bis-(2-methoxy-4-nitro-5-sulfophenyl)-2Htetrazolium-5-carboxanilide (XTT) assay. XTT is a yellow tetrazolium salt cleaved by the mitochondrial dehydrogenase in metabolically active cells to form an orange formazan dye. The formazan dye was measured in an optimal spectrophotometric range of 450-500 nm. All assays were performed in 96-well tissue culture plates coated with $0.1 \mathrm{mg} / \mathrm{ml}$ poly-L-ornithine. Cells were seeded onto prewetted scaffolds. As a positive control, a scaffold which was not integrated with microspheres was used. At the end of the determined time, $50 \mu \mathrm{l}$ of $\mathrm{XTT}$ was added into to the wells and they were kept at $37^{\circ} \mathrm{C}$ for $4 \mathrm{~h}$. Finally, the scaffolds were removed from wells and photometric measurement was carried out at $450 \mathrm{~nm}$. All experiments were repeated a minimum of three times with assays performed in triplicate.

\subsubsection{Differentiation of $P C_{12}$ cell on macroporous $N F$ gelatine scaffold integrated with the NGF-loaded alginate microspheres}

For NGF-induced differentiation studies, complete culture medium was replaced with starvation medium which consisted of RPMI 1640 containing 1\% horse serum. Cells $(30,000)$ were seeded onto the empty scaffold and the scaffold integrated with alginate microspheres (both scaffolds were $200 \mathrm{~mm}^{3}$ in volume) after culturing in starvation medium for $24 \mathrm{~h}$. As a positive control, $50 \mathrm{ng} / \mathrm{ml}$ of NGF was added to the culture medium of scaffold incorporated with empty alginate microspheres. The medium was changed every 2 days and at the end of the $4^{\text {th }}$ and $10^{\text {th }}$ days the scaffolds were removed from the wells and washed in phosphate-buffered saline, fixed with $2.5 \%$ glutaraldehyde for $20 \mathrm{~min}$, dehydrated in a series of graded concentrations of ethanol (30\%, 50\%, 70\%, $80 \%, 90 \%, 100 \%$ ) each for $5 \mathrm{~min}$ and vacuum-dried. Dehydrated constructs were cut, coated with gold and examined in SEM.

\subsection{Statistical analysis}

The significant differences between the groups were evaluated by ANOVA analysis by Tukey's method with 95\% confidence interval. The results were presented as mean \pm standard deviation, which were calculated from at least three independent experiments. 


\section{Results and Discussion}

\subsection{Preparation and characterization of interconnected macroporous NF scaffolds}

Thermally induced phase separation technique consists of polymer dissolution, phase separation, gelation, solvent extraction, freezing and freeze-drying. Under a certain temperature, homogeneous polymer solutions separate into polymer-rich and polymer-lean phases. After removal of the solvent, a 3D porous structure can be obtained from the solidified polymer-rich phase (Kim and Lee 2007). In this study, gelatine was dissolved in an ethanol/water solvent mixture, where ethanol is a nonsolvent of gelatine and adjusts the interaction between gelatine and solvent molecules resulting in the formation of NF structures, as demonstrated by Liu and Ma (2009). Based on this finding, a fixed 50/50 ( $/ / \mathrm{v})$ ethanol/water mixture ratio was used in all experiments to prepare 5\% $(w / v), 7.5 \%$ $(w / v)$ and $10 \%(w / v)$ gelatine concentrations. When gelatine concentration was $5 \%$, a bead-like scaffold structure was observed as shown in Figure 1A indicating that phase separation occurred in the metastable region (Figure 1B). Nam and Park (1999) have shown that at the low polymer concentration end of the metastable region, powder-like polymer solid is obtained as a result of droplets of polymer-rich phase dispersed in the polymer-lean phase. By increasing gelatine concentration from $5 \%$ to $7.5 \%$ and $10 \%$, characteristic NF scaffold structures (well-interconnected nanofibres with small uniform pores) were observed as a result of phase separation that occurred in the spinodal region (Figure 1 C, D). The average diameter and length of the fibres in the scaffolds were determined as $501 \mathrm{~nm}$ and $1267 \mathrm{~nm}$ for the $7.5 \%$ gelatine concentration and $528 \mathrm{~nm}$ and $4390 \mathrm{~nm}$ for the $10 \%$ gelatine concentration. The fiber sizes of both NF scaffolds mimic the size of natural collagen fiber bundles that vary in diameter from 50 to $500 \mathrm{~nm}$. To facilitate fluid flow for nutrient and metabolite waste transport and to support migration and differentiation of cells, macropores were created using paraffin spheres with different sizes (250-425 $\mu \mathrm{m}$ and 425-600 $\mu \mathrm{m})$. The assembly was exposed to heat treatment for $200 \mathrm{~min}$ and $400 \mathrm{~min}$, resulting in high interconnectivity between the pores (Figure 2A, B). Gelatine is a water-soluble polymer; hence, its mechanical stability was improved by crosslinking using a combination of carbodiimide with NHS. The degree of crosslinking was optimized to protect the NF structure in the scaffold (see Supporting Information).

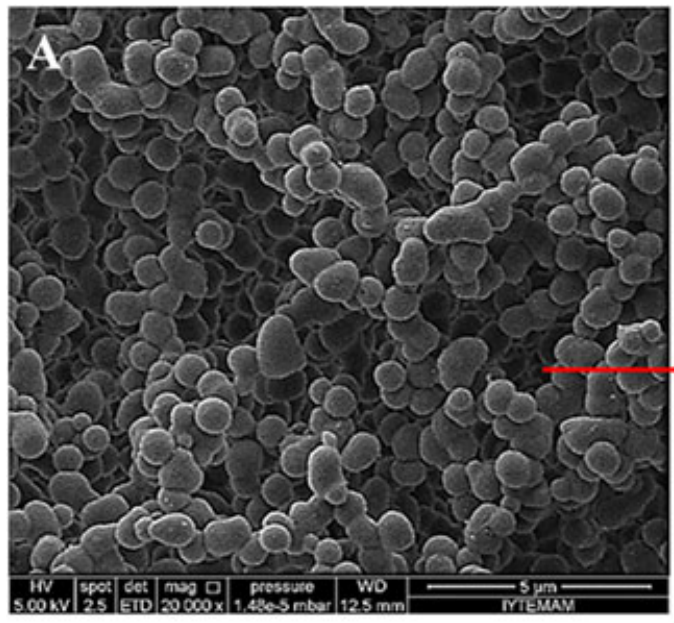

\section{B}
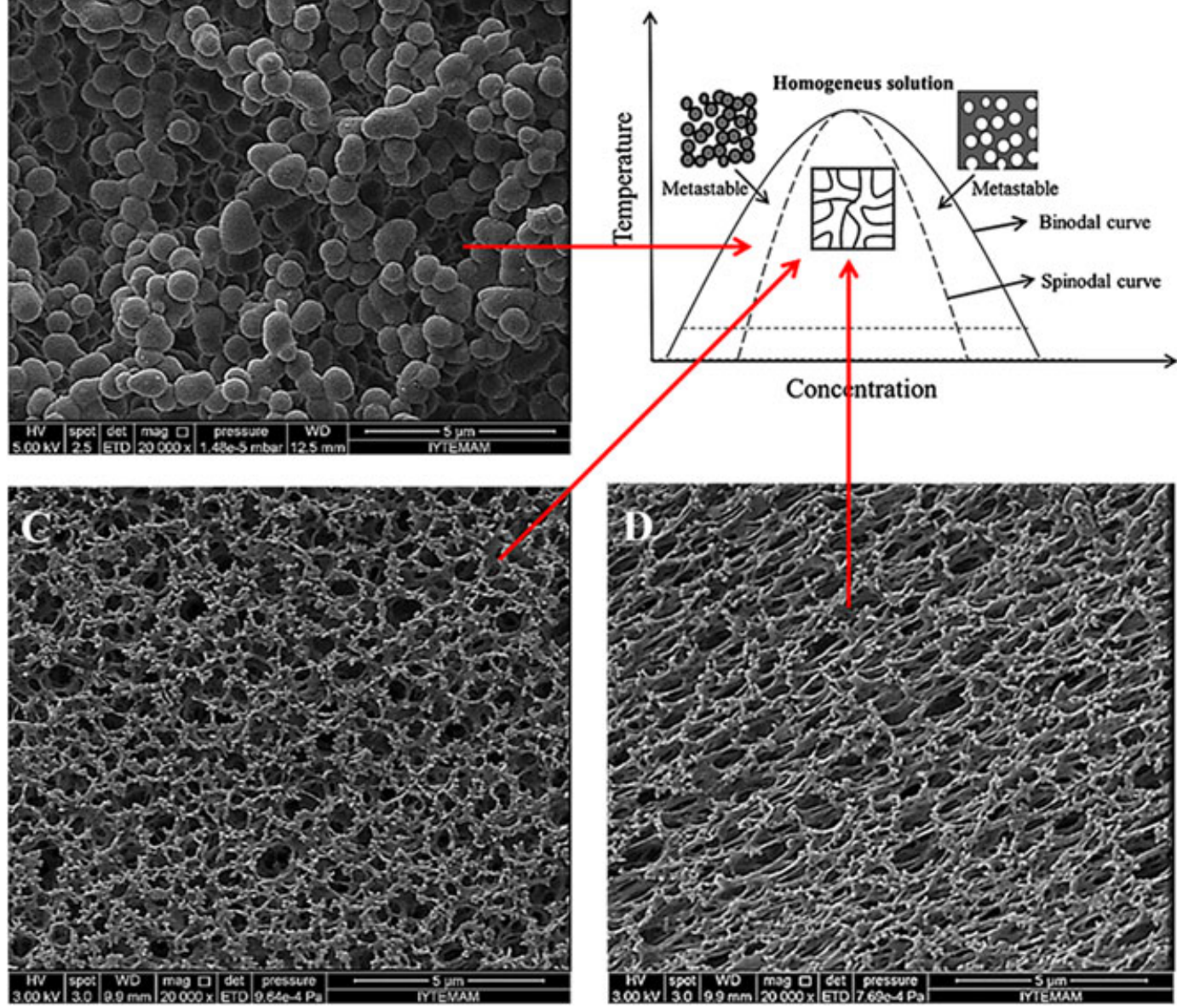

Figure 1. Scaffolds prepared from $5 \%(A), 7.5 \%$ (C) and $10 \%$ (D) gelatine solution $\times 5000$. (B) Schematic phase diagram of polymer solution 
The elastic modulus of the scaffolds was considered as the major design criterion in optimizing preparation conditions, since colonization, migration, differentiation of neural cells (Leipzig and Shoichet 2009; Saha et al., 2008) and altered neurite formation and trajectory (Balgude et al., 2001; Jiang et al., 2008) were found to be influenced by matrix stiffness. Figure 3 shows that all of the scaffolds displayed a typical compressive stressstrain curve; a characteristic profile of the hydrated scaffolds with open-cell foams. In the stress-strain diagram, linear elastic, collapse plateaus and densification regimes were observed with a transition from the linear elastic to collapse plateau region at approximately 5\% strain. Compressive modulus values were calculated from the slope of the linear elastic region and are listed in Table 1. As expected, the stiffness of the scaffolds increased by increasing gelatine concentration from $7.5 \%$ to $10 \%$. By contrast, preheating time had an influence only on the modulus of the scaffold prepared from $10 \%$ gelatine concentration. It was seen that the scaffold prepared from $7.5 \%$ gelatine solution with 250-425 $\mu \mathrm{m}$ sized paraffin spheres that were exposed to $200 \mathrm{~min}$ of heat treatment, had a modulus of $1.2 \pm 0.4 \mathrm{kPa}$, similar to that of brain tissue $(0.5-1 \mathrm{kPa})$ (Pettikiriarachchi et al., 2010). Thus, these conditions were chosen as optimum conditions to prepare suitable scaffolds for brain tissue engineering applications and used in further studies.

\subsection{Preparation and characterization of NGF loaded alginate microspheres}

NGF plays a critical role in the regeneration of neural cells. However, appropriate techniques are needed to incorporate it into scaffolds due to its short half-life (Tria et al., 1994). Target cells respond to NGF when the concentration is above a certain threshold level (Barde 1989). Thus, an optimal delivery system is required to

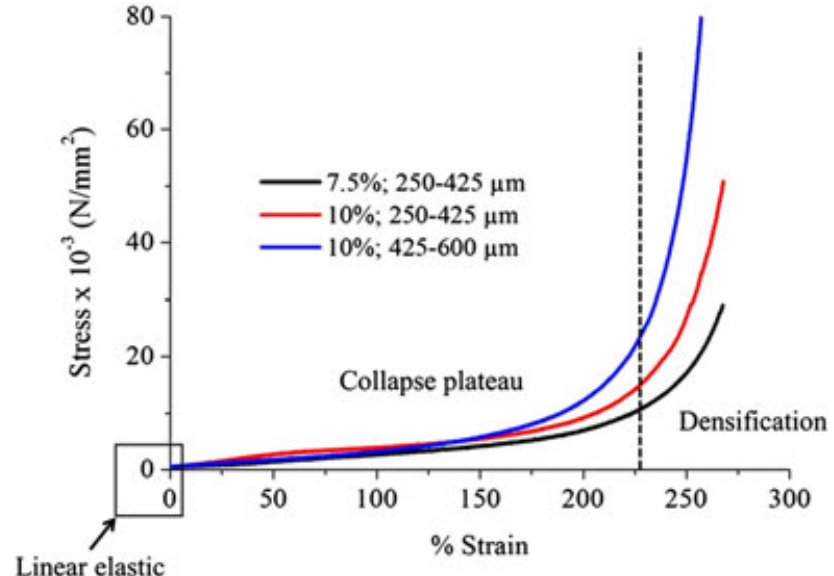

Figure 3. Stress-strain curves from the mechanical test of nanofibrous scaffolds

Table 1. Compressive modulus $(\mathrm{kPa})$ of scaffolds prepared with $7.5 \%$ and $10 \%$ gelatine concentrations

\begin{tabular}{llll}
\hline $\begin{array}{l}\text { Gelatine } \\
\text { concentration }\end{array}$ & $\begin{array}{c}\text { Diameter of } \\
\text { paraffin spheres }\end{array}$ & \multicolumn{2}{c}{ Preheating time } \\
\cline { 3 - 4 } & & $200 \mathrm{~min}$ & $400 \mathrm{~min}$ \\
\hline $7.5 \%$ & $250-425 \mu \mathrm{m}$ & $1.2 \pm 0.4$ & $2.5 \pm 1.7$ \\
$10 \%$ & $425-600 \mu \mathrm{m}$ & $3.3 \pm 0.9$ & $3.8 \pm 1.2$ \\
& $250-425 \mu \mathrm{m}$ & $4.8 \pm 0.3$ & $6.2 \pm 0.4$ \\
& $425-600 \mu \mathrm{m}$ & $9.0 \pm 2.5$ & $5.7 \pm 0.3$ \\
\hline
\end{tabular}

achieve controlled release of NGF. Although direct adsorption of free NGF on the scaffold might be the easiest way for its delivery, desired biological effects cannot be easily achieved due to loss of activity during adsorption and the short duration of release. In addition, direct immobilization may alter the structure, degradation and mechanical properties of the scaffolds. To overcome these disadvantages, NGF has been encapsulated in alginate microspheres. Alginate has been chosen since encapsulation can be carried out at a mild room temperature without using organic solvents and
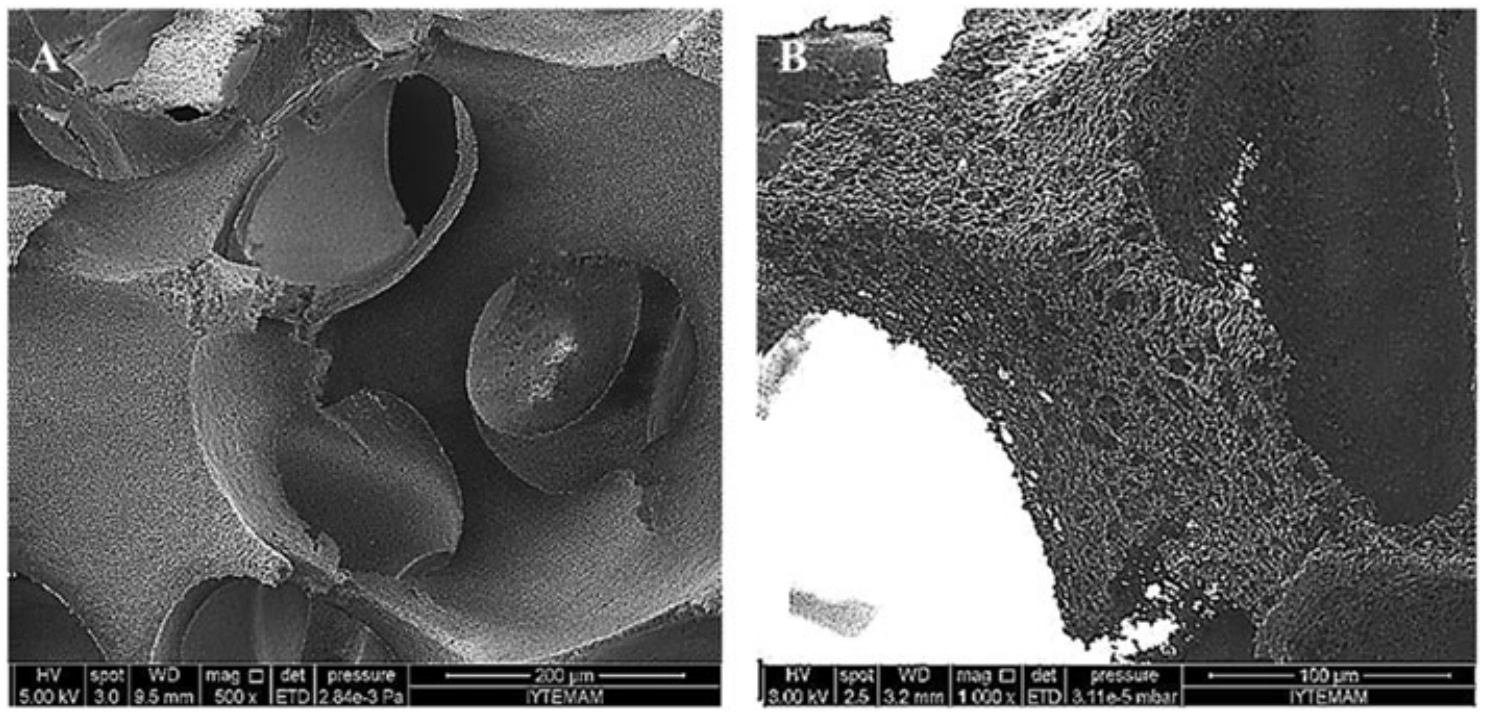

Figure 2. Nanofibrous scaffolds prepared with $10 \%$ gelatine concentration, $200 \mathrm{~min}$ preheating time and $425-600 \mu \mathrm{m}$ sized paraffin spheres 
dissolution, and biodegradation of the system takes place under physiological conditions. NGF-loaded alginate microspheres were produced by water-in-oil emulsification technique using a mixture of hydrophilic and lipophilic surfactants, Tween 80 and Span 80. Ciofani et al., (2008) prepared alginate microspheres with a similar method using only Tween 85 as a surfactant and reported $70 \%$ yield (ratio of mass of microspheres obtained to the mass of polymer used in the emulsion) while our yield was $82.5 \%$. Although hydrophilic lipophilic balances (HLB) for the mixture of Span 80 and Tween 80 (HLB: 10.7) and pure Tween 85 (HLB: 11) were similar, the higher yield obtained with a blend of surfactants can be attributed to better dispersion and solubilization into continuous phase (Porras et al., 2004).

The microspheres that were synthesized with this technique had a smooth structure with a spherical shape (Figure 4A-D) and they were attached to the scaffolds
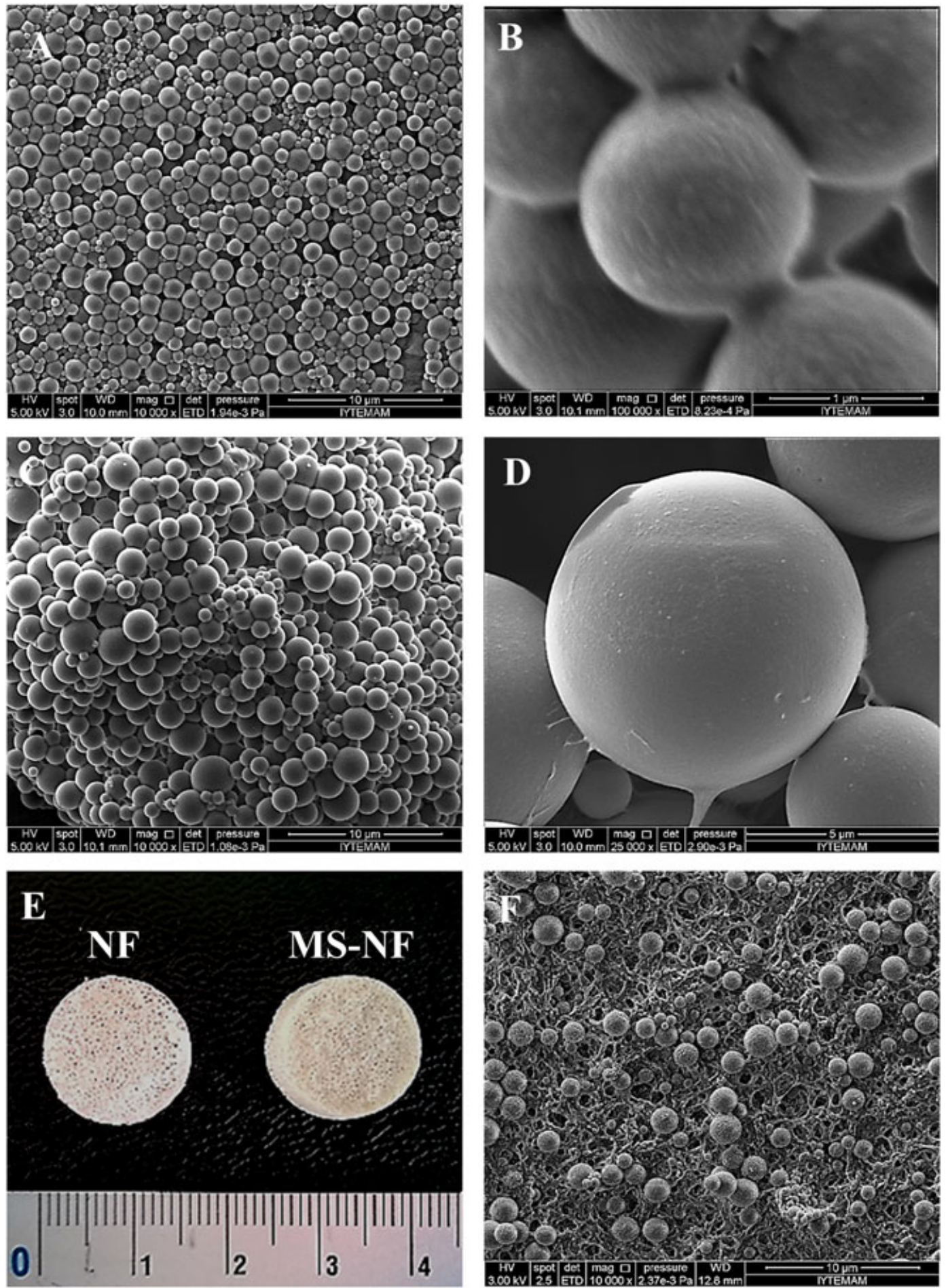

Figure 4. SEM images of (A, B) $0.1 \%(w / v)(C, D) 1 \%(w / v)$ alginate microspheres. (E) General appearance of nanofibrous scaffolds before (NF) and after (MS-NF) microsphere attachment. (F) SEM image of MS-N 
with a high efficiency (94\%). As seen in Figure 4E, the general appearance of the NF scaffolds was white and opaque and attachment of alginate microspheres did not cause a morphological or structural change in the scaffolds (Figure 4F). Uniform distribution of alginate microspheres was achieved due to seeding of particles from both sides of the scaffold (Figure S3). Average diameter of the unloaded microspheres increased from $1.72 \pm 0.11$ to $5.2 \pm 0.51 \mu \mathrm{m}$ with the increased alginate concentration (Figure 5). NGF loading into $0.1 \%$ alginate microspheres did not change the microsphere size, while $1 \%$ alginate particles reduced in size as loading level increased. This can be attributed to the presence of a higher number of free carboxylate groups and higher negative charge in $1 \%$ alginate microspheres causing a stronger interaction with positively charged groups in NGF, yielding more compact particles. High charge density and large size of $1 \%$ alginate microspheres allowed $100 \%$ encapsulation of $1 \mu \mathrm{g}$ NGF while encapsulation efficiency for $0.1 \%$ alginate microspheres was $85 \%$. High encapsulation efficiency is a desired property for cost-effective utilization of growth factors.

\subsubsection{NGF release from free and attached alginate microspheres}

To determine release mechanism, the release of NGF from free microspheres directly added into the release medium was first measured and the results are shown in Figure 6 A. In all cases, burst release was observed in the first $5 \mathrm{~h}$ due to diffusion of water into alginate gels and release of loosely attached NGF on the surface. At later times, the release was controlled by diffusion of NGF through the alginate matrix and dissolution of microspheres caused by bulk erosion and decrosslinking. As expected, slower NGF release was observed from the $1 \%$ alginate particles due to their larger sizes compared to the size of the $0.1 \%$ alginate microspheres. In addition, higher negative charge on the $1 \%$ alginate particles makes the removal of $\mathrm{Ca}^{2+}$ ions, and hence decrosslinking in the structure, difficult due to strong electrostatic interaction between the alginate and positively-charged NGF (Wee and Gombotz 1998). Consequently, fast release of NGF was prevented. Alginate can make a strong ionic

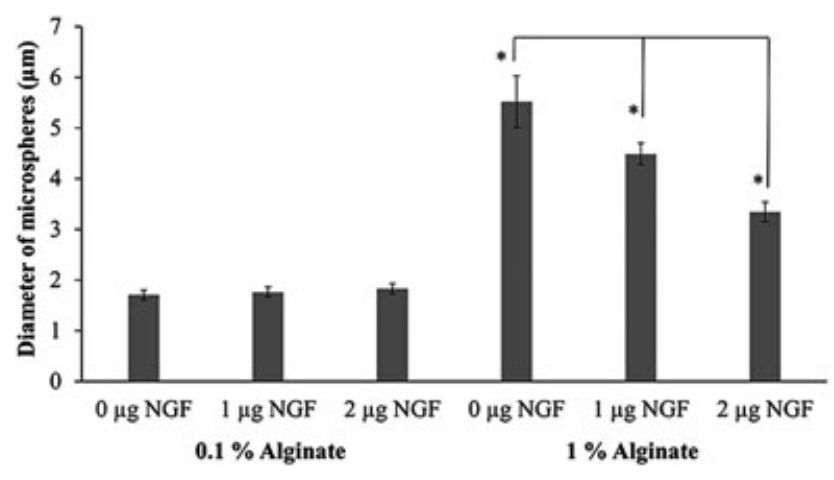

Figure 5. Effect of alginate concentration and NGF loading on the size of microspheres $p<0.05$ interaction with polycations which can be used to stabilize the integrity of gel and to delay protein release (Smidsrod 1973). This property of alginate was used and PLL was selected as the polycation, to coat the alginate microspheres. As seen from Figure 6A, coated particles showed a more sustained release profile as a result of modified surface properties and additional barrier against NGF diffusion, in addition, coating on smaller microspheres (prepared with $0.1 \%$ alginate) was found more effective in delaying NGF release. It has previously been reported that binding of PLL to the alginate is followed by the diffusion of PLL into the alginate network forming a complex membrane on the surface of the microspheres (Thu et al., 1996). This semipermeable membrane decreases both the burst effect and the release rate (Lemoine et al., 1998). The alginate-PLL interactions are governed by the stoichiometric ratio of the charged groups, suggesting that the number of $\mathrm{NH}_{3}^{+}$groups in PLL is not sufficient to interact with most of the $\mathrm{COO}^{-}$ groups in the $1 \%$ alginate; hence, release from PLL coated $1 \%$ alginate microspheres is not significantly delayed.

Figure 6B shows that immobilization of the microspheres reduced the release of NGF, likely due to diffusional resistance of the scaffold and reattachment of the released NGF to the surface of the scaffold (Wei et al., 2006). The release patterns of free and attached microspheres were found to be different. Total amount of NGF released from both free microspheres at the end of $100 \mathrm{~h}$ was similar (Figure 6A). By contrast, NGF released from $1 \%$ immobilized microspheres was 1.4 times lower compared to the amount released from the $0.1 \%$ attached particles due to the presence of more negatively charged groups in $1 \%$ alginate causing redeposition of released NGF on the surface of the particles. The release of NGF from the scaffolds integrated with PLL coated $0.1 \%$ alginate microspheres was also measured (Figure 6B). PLL coating reduced the release rate of NGF from $1040 \mathrm{pg} / \mathrm{h}$ to $680 \mathrm{pg} / \mathrm{h}$ within 10 days. The results in Figure 6B suggest that the release profile of NGF can be adjusted by the alginate concentration or PLL coating to achieve the desired release rate.

\section{3. $\mathrm{PC}_{12}$ cell proliferation and differentiation}

\subsubsection{PC12 cell proliferation on macroporous NF gelatine scaffolds integrated with empty alginate microspheres}

Proliferation of seeded cells in the scaffold is required to enable new tissue development. In the preliminary study, cell proliferation on petri dishes and the gelatine scaffolds were compared. In these experiments, the same number of cells was seeded into the gelatine scaffold and petri dishes of the same size. The results in Figure S4 have shown that until the $9^{\text {th }}$ day, the rate of cell growth in 2D (petri dish) and 3D (scaffold) cell culture media was similar. However, at the end of 12 days, the cell population on the scaffold was significantly higher than 

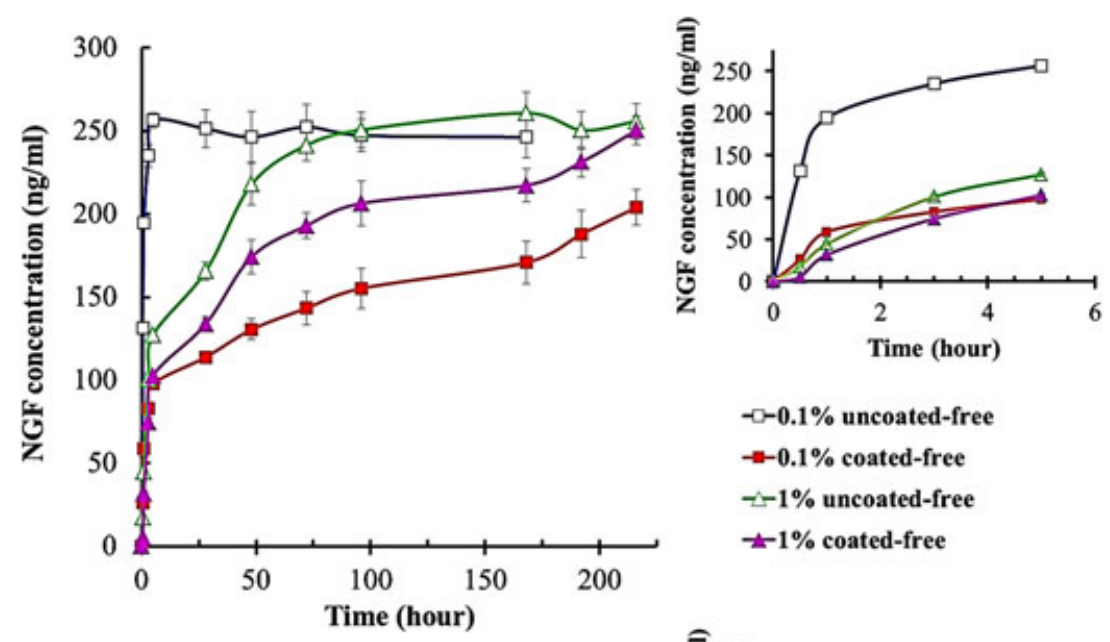

$\rightarrow-0.1 \%$ uncoated-free

$-0.1 \%$ coated-free

$-1 \%$ uncoated-free

$\pm-1 \%$ coated-free

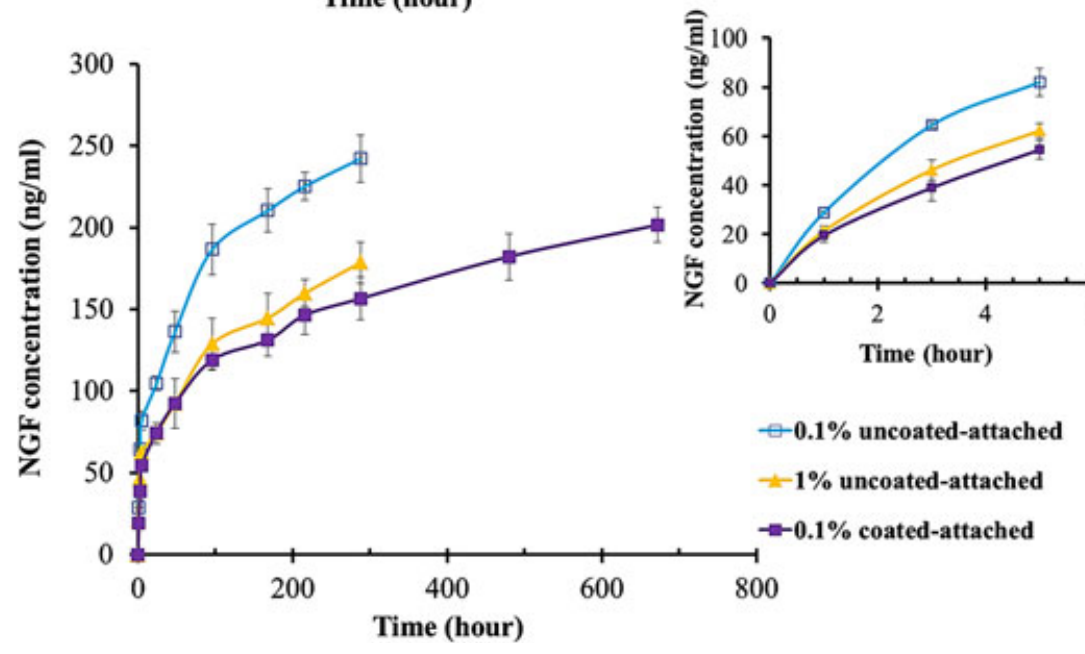

Figure 6. Release of NGF from (A) uncoated and coated free alginate microspheres and (B) the scaffolds integrated with alginate microspheres

those on petri dish, which clearly indicated the advantage of the 3D environment on cell proliferation.

As seen from Figure 7, at the end of 10 days, the number of cells in the empty scaffold and the scaffold integrated with $0.1 \%$ unloaded alginate microspheres were similar $(p>0.05)$. The lower absorbance value, in $0.1 \%$ alginate group at the $6^{\text {th }}$ day compared to empty scaffold, was due to the presence of impurities in the alginate which were removed during the exchange of cell culture medium; thus, the cell population significantly

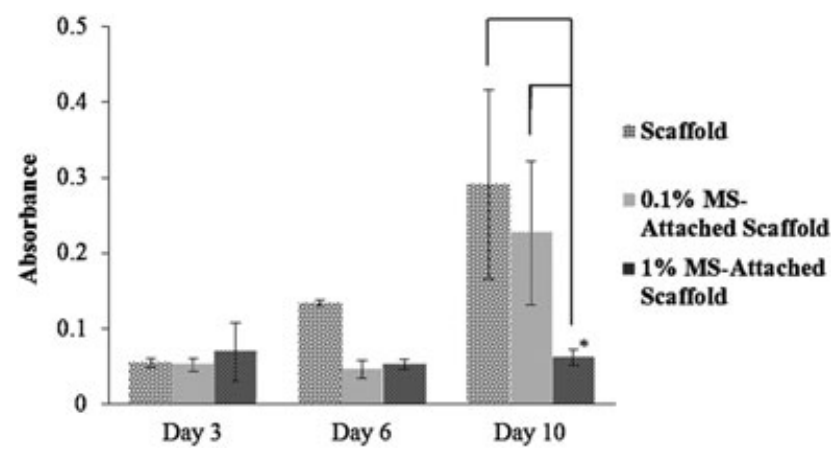

Figure 7. Absorbance value of PC12 cells cultured on empty (Scaffold) and alginate microspheres attached (MS-Attached Scaffold) scaffolds. $p<0.05$ increased from $6^{\text {th }}$ to $10^{\text {th }}$ day. It was found that $1 \%$ unloaded alginate particles in the scaffold inhibited the proliferation of cells. The inhibition is due to a high alginate concentration which decreased the $\mathrm{pH}$ of the cell culture medium from 7.2 to 6.7 at the end of $24 \mathrm{~h}$ and this stopped proliferation process as also reported by Mackenzie et al. (1961). A similar inhibition effect was also reported for fibroblast and Schwann cells where the proliferation rates were lower when these cells were cultured in a high concentration of alginate solution compared to the proliferation rates observed in the low concentration alginate solution (Bohari et al., 2011; Cao et al., 2012).

\subsubsection{PC12 cell differentiation on macroporous NF gelatine scaffold integrated with NGF loaded alginate microspheres}

The differentiation of PC12 cells was demonstrated using SEM images taken at the end of 4 and 10 days after seeding the cells. Figures $8 \mathrm{~A}$ and $8 \mathrm{~B}$ show that the shapes of PC12 cells attached on the empty scaffolds, incubated with and without free NGF, are similar, which indicated that $150 \mathrm{ng} / \mathrm{ml}$ of free NGF added within 4 days was not enough to initiate the differentiation of the cells. Similarly, at the end of 4 days no differentiation was 

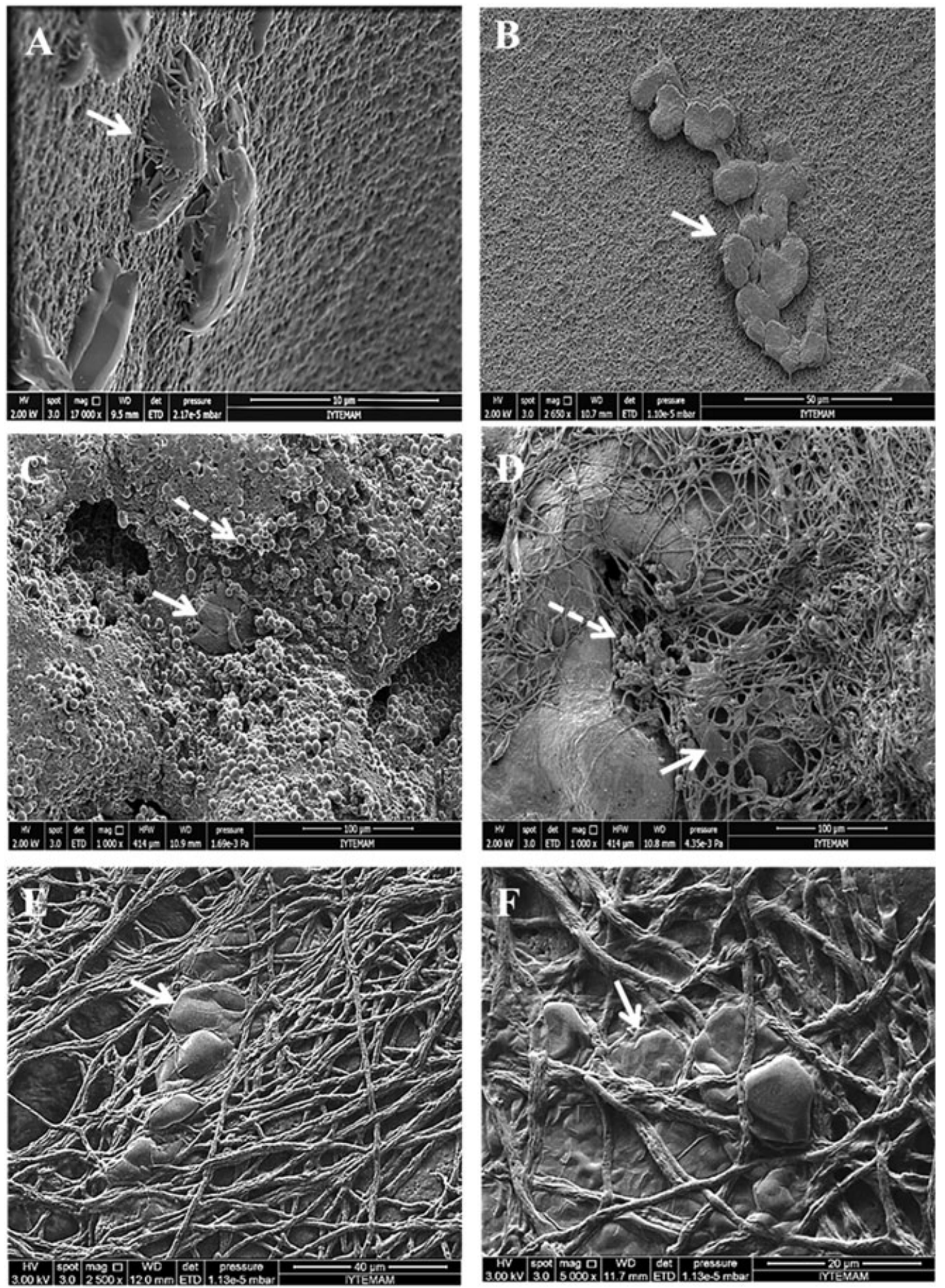

Figure 8. Fourth day SEM images of PC12 cells seeded on (A) empty scaffold; (B) scaffold treated with $50 \mathrm{ng} / \mathrm{ml} / 2$ days of free NGF, (C) the scaffold attached with NGF loaded $1 \%$ alginate microspheres and (D-F) $0.1 \%$ alginate microspheres. Solid and dashed arrows indicate PC12 cells and alginate microspheres attached on the scaffold

observed on the scaffold integrated with $1 \%$ alginate microspheres (Figure 8C); however, NGF released from $0.1 \%$ alginate microspheres induced well-organized neurite structure formation (Figures 8D-F). Figure 8D-F correspond to the SEM pictures of the same scaffold integrated with NGF loaded $0.1 \%$ alginate microspheres. The three elements of the construct - scaffold, cells and alginate particles - along with the PC12 cell neurites are shown with arrows (Figure 8D and Figure S5). Due to intense neurite extension, alginate particles cannot be seen in Figures 8E and 8F taken at higher magnification. Although the amounts of free NGF added to the scaffold and NGF released from $0.1 \%$ attached microspheres at the end of 4 days were almost equal $(\sim 150 \mathrm{ng} / \mathrm{ml})$, the absence of neurite extension in the case of direct adsorption of free NGF to the scaffold indicated a loss of NGF activity. All of the scaffolds supported differentiation of PC12 cells at the end of 10 days (Figure 9), however, 

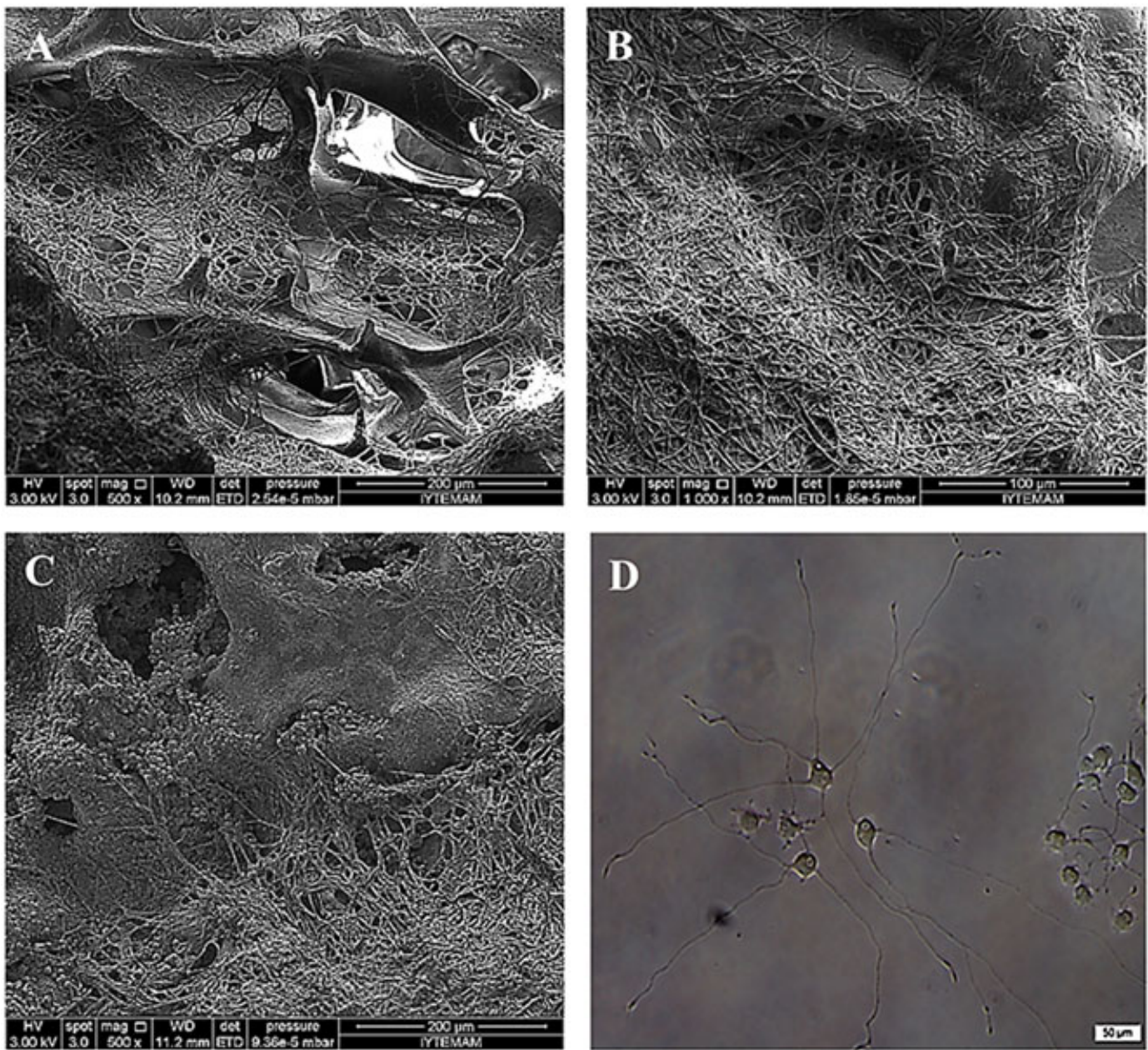

Figure 9. Tenth day SEM images of PC12 cells seeded on the scaffold attached with NGF loaded (A, B) $0.1 \%$, (C) $1 \%$ alginate microspheres. (D) Tenth day phase contrast microscope images of PC12 cells in 2D cell culture flask in starvation medium fed with $50 \mathrm{ng} / \mathrm{ml} / 2$ days of NGF [Colour figure can be viewed at wileyonlinelibrary.com]

the most extensive neurite outgrowth and tissue-like network formation was visualized in the scaffold integrated with $0.1 \%$ microspheres (Figure 9A and 9B). Local $\mathrm{pH}$ in the microenvironment of cells controls not only cell proliferation but also cellular activity (Mackenzie et al., 1961; Taylor 1962). Delayed differentiation of cells in the scaffold in the presence of $1 \%$ alginate microspheres is probably caused by the decrease in $\mathrm{pH}$, which also had an adverse effect on the proliferation of the cells. Another reason for delayed neurite outgrowth is lower NGF level in the microenvironment of the cells since NGF release from $1 \%$ alginate microspheres is slower (Figure 6B). Neurite structure on 3D network (Figure 9A-C) was much more complex and intense than that observed in 2D cell culture medium (Figure 9D). This result showed that NF $3 \mathrm{D}$ scaffolds provided a microenvironment mimicking the extracellular matrix, a higher surface area for growth and migration and protected cells from environmental disturbances. In addition, encapsulating NGF into the alginate microspheres helped in preserving biological activity and maintained, controlled and sustained release of NGF. Consequently, survival and differentiation of the cells in the scaffolds were improved.

\section{Conclusions}

In this study, a new biomimetic gelatine based NF scaffold with NGF-loaded microspheres was prepared for the first time for brain tissue engineering applications. Thermally induced phase separation combined with a porogen leaching method allowed control of porosity, pore size, interpore connectivity, mechanical properties as well as pore morphologies of the scaffolds to promote neural regeneration. The scaffold with a modulus value similar to that of brain tissue was obtained and used for cell proliferation studies. NGF was encapsulated into alginate microspheres with a high loading efficiency without utilizing harsh conditions. Crosslinking of the scaffolds and attachment of the alginate microspheres to the scaffold were accomplished easily in a single step with a very high post seeding efficiency of the microspheres. The release rate of NGF from the microspheres was controlled by the alginate concentration and poly(Llysine) coating. Alginate concentration was found to be critical in controlling the release rate of NGF, as well as proliferation and differentiation of PC12 cells. The scaffolds prepared in this study can also be combined with other neurotrophins such as brain derived neurotrophic 
factor, and neurotrophins-3 and -4. High encapsulation efficiencies of these factors into alginate microspheres are expected since they have similar molecular weights $(13.2-15.9 \mathrm{kDa}$ ) and isoelectrical points (9 or 10) to those of NGF. With interconnected macropore structure for axonal growth and nutrient transport, similar topologic and mechanical properties of brain tissue and capability of controlled release of nerve growth factor, gelatinebased biomimetic scaffolds have great potential as implantable matrixes for the regeneration of destroyed tissues derived from traumatic brain injuries. In future work, the in vivo performance of these scaffolds will need to be investigated.

\section{Conflicts of interest}

The authors have declared that there is no conflict of interest.

\section{Acknowledgements}

This study was supported by The Scientific and Technical Research Council of Turkey (TUBITAK, Grant Number: 112 M568). We also would like to thank Izmir Institute of Technology, Biotechnology and Bioengineering Research and Application Center and Material Research Center for providing experimental support and Professor Brian Carr for his valuable comments.

\section{References}

Balgude AP, Yu X, Szymanski A et al. 2001; Agarose gel stiffness determines rate of DRG neurite extension in 3D cultures. Biomaterials 22(10): 1077-1084.

Barde YA. 1989; Trophic Factors and Neuronal Survival. Neuron 2: 1525-1534.

Bohari SP, Hukins DW, Grover LM. 2011; Effect of calcium alginate concentration on viability and proliferation of encapsulated fibroblasts. Biomed Mater Eng 21(3): 159-170.

Cao N, Chen XB, Schreyer DJ. 2012; Influence of calcium ions on cell survival and proliferation in the context of an alginate hydrogel. ISRN Chem Eng 516461: 1-9.

Chen R, Curran SJ, Curran JM et al. 2006; The use of poly(llactide) and RGD modified microspheres as cell carriers in a flow intermittency bioreactor for tissue engineering cartilage. Biomaterials 27(25): 4453-4460.

Ciofani G, Raffa V, Menciassi A et al. 2008; Alginate and chitosan particles as drug delivery system for cell therapy. Biomed Microdevices 10(2): 131-140.

D'Avanzo C, Aronson J, Kim YH et al. 2015; Alzheimer's in 3D culture: challenges and perspectives. BioEssays 37(10): 1139-1148

Gu F, Amsden B, Neufeld R. 2004; Sustained delivery of vascular endothelial growth factor with alginate beads. $J$ Control Release 96(3): 463-472.

Hermanson GT. 1996; Protein crosslinking method. In Bioconjugate Techniques, Hermanson GT (ed). Academic Press: San Diego, CA: 144-148.

Jiang FX, Yurke B, Firestein BL. 2008; Neurite outgrowth on a DNA crosslinked hydrogel with tunable stiffnesses. Ann Biomed Eng 36(9): 1565-1579.

Kim HD, Lee DS. 2007; Protocol for thermally induced phase separation (TIPS). In A Manual for Biomaterials/Scaffold Fabrication Technology. Khang G, Kim MS, Lee HB (eds). World Scientific: Singapore: $101-110$

Kim YH, Choi SH, D'Avanzo C et al 2015; A 3D human neural cell culture system for modeling Alzheimer's disease. Nat Protoc 10(7): 985-1006.

Leipzig ND, Shoichet MS. 2009; The effect of substrate stiffness on adult neural stem cell behaviour. Biomaterials 30(36): 6867-6878.

Lemoine D, Wauters F, Bouchend'homme S et al. 1998; Preparation and characterization of alginate microspheres containing a model antigen. Int $J$ Pharm 176(1): 9-19.

Liu X, Ma PX. 2009; Phase separation, pore structure, and properties of nanofibrous gelatin scaffolds. Biomaterials 30(25): 4094-4103.
Ma F, Xiao Z, Chen B et al. 2014; Accelerating proliferation of neural stem/progenitor cells in collagen sponges immobilized with engineered basic fibroblast growth factor for nervous system tissue engineering. Biomacromolecules 15(3): 1062-1068.

Ma PX, Choi JW. 2001; Biodegradable polymer scaffolds with well-defined interconnected spherical pore network. Tissue Eng 7(1): 23-33.

Ma PX. 2004; Scaffolds for tissue fabrication. Mater Today 7(5): $30-40$.

Mackenzie CG, Mackenzie JB, Beck P. 1961; The effect of pH on growth, protein synthesis, and lipid-rich particles of cultured mammalian cells. J Biophys Biochem Cytol 9: $141-156$

Mahmood A, Wu H, Qu C et al. 2014; Down-regulation of Nogo-A by collagen scaffolds impregnated with bone marrow stromal cell treatment after traumatic brain injury promotes axonal regeneration in rats. Brain Res 1542: 41-48.

Mak M, Fung L, Strasser JF et al. 1995; Distribution of drugs following controlled delivery to the brain interstitium. J Neuro-Oncol 26(2): 91-102.

Nam YS, TG Park. 1999; Biodegradable polymeric microcellular foams by modified thermally induced phase separation method. Biomaterials 20(19): 1783-1790.

Pettikiriarachchi JTS, Parish CL, Shoichet MS et al. 2010; Biomaterials for brain tissue engineering. Aust J Chem 63: 1143-1154.

Porras M, Solans C, Gonzáleza C et al. 2004; Studies of formation of W/O nano-emulsions. Colloids Surf A Physicochem Eng Asp 249(1-3): 115-118.

Qu C, Mahmood A, Liu XS et al. 2011; The treatment of TB with human marrow stromal cells impregnated into collagen scaffold: functional outcome and gene expression profile. Brain Res 1371: 129-139.

Qu C, Xiong Y, Mahmood A et al. 2009; Treatment of traumatic brain injury in mice with bone marrow stromal cell-impregnated collagen scaffolds. J Neurosurg 111(4): 658-665.

Saha K, Keung AJ, Irwin EF et al. 2008; Substrate modulus directs neural stem cell behavior. Biophys J 95(9): 4426-4438.

Schmidt CE, Leach JB. 2003; Neural tissue engineering: strategies for repair and regeneration. Annu Rev Biomed Eng 5: 293-347.

Simão D, Pinto C, Piersanti S et al. 2015; Modeling human neural functionality in vitro: three-dimensional culture for dopaminergic differentiation. Tissue Eng Part A 21(3-4): 654-668.

Smidsrod O. 1973; The relative extension of alginates having different chemical composition. Carbohydr Res 27(1): 107-118.

Spires T, Grote H, Varshney N et al. 2004; Environmental enrichment rescues protein deficits $\mathrm{i}-\mathrm{n}$ a mouse model of Huntington's disease, indicating a possible disease mechanism. J Neurosci 24(9): 2270-2276.

Tam RY, Fuehrmann T, Mitrousis N et al. 2014; Regenerative therapies for central nervous system diseases: a biomaterials approach. Neuropsychopharmacology 39(1): 169-188.

Tang-Schomer MD, White JD, Tien LW et al. 2014; Bioengineered functional brain-like cortical tissue. Proc Natl Acad Sci U S A 111(38): 13811-13816.

Taylor AC. 1962; Responses of cells to pH changes in the medium. J Cell Biol 15(2): 201-209.

Thu B, Bruheim P, Espevik Tet al. 1996; Alginate polycation microcapsules. I. Interaction between alginate and polycation. Biomaterials 17(10): 1031-1040.

Tria MA, Fusco M, Vantini G et al. 1994; Pharmacokinetics of nerve growth factor (NGF) following different routes of administration to adult rats. Exp Neurol 127(2): 178-183.

Vukelja SJ, Anthony SP, Arseneau JC et al. 2007; Phase 1 study of escalating-dose OncoGel (ReGel/paclitaxel) depot injection, a controlled-release formulation of paclitaxel, for local management of superficial solid tumor lesions. Anti-Cancer Drugs 18(3): 283-289.

Wee S, Gombotz WR. 1998; Protein release from alginate matrices. Adv Drug Deliv Rev 31(3): 267-285.

Wei G, Jin Q, Giannobile WV et al. 2006; Nano-fibrous scaffold for controlled delivery of recombinant human PDGF-BB. J Control Release 112(1): 103-110.

Wei G, Jin Q, Giannobile WV et al. 2007a; The enhancement of osteogenesis by nano-fibrous scaffolds incorporating rhBMP-7 nanospheres. Biomaterials 28(12): 2087-2096. Wei G, Ma PX. 2008; Nanostructured biomaterials for regeneration. Adv Funct Mater 18(22): 3566-3582.

Wei YT, Tian WM, Yu X et al. 2007b; Hyaluronic acid hydrogels with IKVAV peptides for tissue repair and axonal regeneration in an injured rat brain. Biomed Mater 2(3): S142-S146.

Westphal M, Hilt DC, Bortey E et al. 2003; A phase 3 trial of local chemotherapy with biodegradable carmustine (BCNU) wafers (Gliadel wafers) in patients with primary malignant glioma. Neuro-Oncology 5(2): 79-88.

\section{Supporting information}

Additional Supporting Information may be found online in the supporting information tab for this article.

Figure S1. Effect of EDC/COOH free $(\mathrm{mole} / \mathrm{mole}$ ) ratio on absorbance value Figure S2. NF-scaffold crosslinked with EDC: $\mathrm{COOH}_{\text {free }}$ ratio of (2: 1) (mole/mole) 
Figure S3. (A) SEM images of cross-section of the scaffold integrated with alginate microspheres, (B) $200 \times$, (C) $500 \times$, (D) $1000 \times$, (E) $2000 \times$.

Figure S4. Absorbance value of PC12 cells cultured on macroporous nanofibrous gelatine scaffold (3D) and petri dishes of the same size (2D) $p<0.05$.

Figure S5. Fourth day SEM images of PC12 cells seeded on the scaffold attached with NGF loaded 0.1\% alginate microspheres. Arrows indicate scaffold surface, microspheres, PC12 cells and cell neurites. 